\title{
The dark side of Microphytobenthos: diel dynamics of nitrate respiration in microbial mats and sediments.
}

\author{
ELISA MERZ ${ }^{1}$, GREGORY J. DICK ${ }^{2}$, DIRK DE BEER ${ }^{1}$, \\ GAUTE LAVIK $^{1}$, HANNAH K. MARCHANT ${ }^{1}$ AND JUDITH$$
\text { M. } \operatorname{KLATT}^{1,2}
$$ \\ ${ }^{1}$ Max-Planck-Institute for Marine Microbiology \\ ${ }^{2}$ University of Michigan \\ Presenting Author: emerz@mpi-bremen.de
}

Benthic diatoms are among the main primary producers globally and their role as phototrophs is well studied. However, some benthic diatoms can also respire internally stored nitrate via DNRA (dissimilatory reduction of nitrate to ammonium) in the absence of light and oxygen. We studied the nitrate respiration strategies of different cultivated benthic diatom species and revealed a wide spectrum of responses to darkness and anoxia. Intriguingly, diatoms kept in aerobic culture for years, took several days to respire nitrate when first exposed to these conditions. However, subsequent repeated switches between oxia and anoxia shortened the induction times for DNRA. While it has been assumed that diatoms utilise nitrate respiration largely as a survival strategy upon, for example, burial events, these results suggest that diatoms might be capable of utilising DNRA on a regular basis in situ. Indeed, using microsensors and stable isotope probing, we found that diatoms in microbial mats perform DNRA on a daily basis, and that it was coupled to their diel migration into dark and anoxic sediments. As a consequence, diatoms have the potential to fundamentally shape the bioavailable N-budget of the illuminated redox-dynamic sediment systems. 